\title{
States and Measures on Hyper BCK-Algebras
}

\author{
Xiao-Long Xin and Pu Wang \\ Department of Mathematics, Northwest University, Xian 710069, China \\ Correspondence should be addressed to Xiao-Long Xin; xlxinxa@126.com \\ Received 20 November 2013; Accepted 1 February 2014; Published 13 March 2014 \\ Academic Editor: Baolin Wang
}

Copyright (C) 2014 X.-L. Xin and P. Wang. This is an open access article distributed under the Creative Commons Attribution License, which permits unrestricted use, distribution, and reproduction in any medium, provided the original work is properly cited.

\begin{abstract}
We define the notions of Bosbach states and inf-Bosbach states on a bounded hyper BCK-algebra $(H, o, 0, e)$ and derive some basic properties of them. We construct a quotient hyper BCK-algebra via a regular congruence relation. We also define a o-compatibled regular congruence relation $\theta$ and a $\theta$-compatibled inf-Bosbach state $s$ on $(H, \circ, 0, e)$. By inducing an inf-Bosbach state $\widehat{s}$ on the quotient structure $H /[0]_{\theta}$, we show that $H /[0]_{\theta}$ is a bounded commutative BCK-algebra which is categorically equivalent to an $\mathrm{MV}$-algebra. In addition, we introduce the notions of hyper measures (states/measure morphisms/state morphisms) on hyper BCKalgebras, and present a relation between hyper state-morphisms and Bosbach states. Then we construct a quotient hyper BCKalgebra $H / \operatorname{Ker}(m)$ by a reflexive hyper BCK-ideal $\operatorname{Ker}(m)$. Further, we prove that $H / \operatorname{Ker}(m)$ is a bounded commutative BCK-algebra.
\end{abstract}

\section{Introduction}

The theory of hyper structures (also called multialgebras) was introduced in 1934 by Marty [1] at the 8th Congress of Scandinavian Mathematicians. Then several researchers have worked on this new field and developed it. Corsini studied the theory of Hypergroups; see [2, 3]. Krasner [4] introduced the notion of hyperrings and hyperfields. Massouros [5] introduced the theory of hypercompositional structures into the theory of automata. Jun et al. [6] introduced the concept of hyper BCK-algebras which is a generalization of BCKalgebras and studied some properties of them. They also introduced the notions of hyper BCK-ideals, weak/strong hyper BCK-ideals, and reflexive hyper BCK-ideals and discussed the relations among these notions. From then on, a lot of literatures about hyper BCK/BCI-algebras appear; see [7-11].

MV-algebras entered mathematics just 50 years ago due to Chang [12], but the notion of states for MV-algebras was introduced by Mundici [13] in 1995 as averaging of the truthvalue in Łukasiewicz logic. BL-algebras were introduced in the 1990s by Hájek as the equivalent algebraic semantics for its basic fuzzy logic. Ciungu et al. [14] defined a state-operator and a strong state-operator for a BL-algebra and proved some basic properties of them. Liu [15] studied the existence of Bosbach states and Riecan states on finite monoidal $t$ - norm based algebras (MTL-algebra for short) and gave some examples to show that there exist MTL-algebras having no Bosbach states and Riecan states.

Dvurečenskij [16] introduced measures and states on BCK-algebras and showed that the set of elements of measure 0 is an ideal and the corresponding quotient BCK-algebra is commutative with a lifted original measure. Corina Ciungu and Dvurečenskij [17] extended the notions of measures and states, which were presented in the paper of Dvurečenskij and Pulmannová [18] to the case of pseudo-BCK-algebras. They also studied similar properties and proved that the notion of states in the sense of Dvurečenskij and Pulmannová [18] coincides with the Bosbach state.

At present, the state theories were set up in various algebraic structures. So far, we have not found research literatures about the state theory on hyper structures. In this paper, we mainly introduce and study the state theory on hyper BCK-algebras.

The paper is organized as follows. In Section 2, we recall some basic notions and some results of hyper BCKalgebras. Then we induce two new operations " $\wedge$ " and "--" by the operation "o" on hyper BCK-algebras and investigate some properties of them. We also present a relation between hyper BCK-algebras and MV-algebras. In Section 3, we define a Bosbach state and an inf-Bosbach state on a bounded hyper BCK-algebra and discuss some of their basic 
properties. In Section 4, we study inf-Bosbach states on quotient hyper BCK-algebras. In Section 5, we define hyper measure, hyper states, hyper measure-morphisms, and hyper state-morphisms on hyper BCK-algebras and obtain some interesting results.

\section{Preliminaries}

In this section, we gather some basic notions and properties relevant to hyper BCK-algebras which we need in the sequel.

Let $H$ be a nonempty set with a hyperoperation "о." For any two subsets $A$ and $B$ of $H$, by $A \circ B$ we mean the set $\bigcup_{a \in A, b \in B} a \circ b$. Hereafter we denote $x \circ y$ instead of $x \circ\{y\}$, $\{x\} \circ y$, or $\{x\} \circ\{y\}$.

Definition 1 (see [10]). Let $H$ be a nonempty set endowed with a hyperoperation "o" and a constant 0 . If $(H, \circ, 0)$ satisfies the following axioms: for all $x, y, z \in H$,

$(\mathrm{HK} 1)(x \circ z) \circ(y \circ z) \ll x \circ y$,

$(\mathrm{HK} 2)(x \circ y) \circ z=(x \circ z) \circ y$,

(HK3) $x \circ H \ll\{x\}$,

(HK4) $x \ll y$ and $y \ll x$ imply $x=y$,

then $H$ is called a hyper BCK-algebra, where $x \ll y$ is defined by $0 \in x \circ y$ and for any nonempty subsets $A, B$ of $H, A \ll B$ is defined by for all $a \in A$; there exists $b \in B$ such that $a \ll b$.

Example 2 (see [10]). We define an operation “o” on $H=$ $[0, \infty)$ by

$$
x \circ y= \begin{cases}{[0, x],} & \text { if } x \leq y \\ (0, y], & \text { if } x>y \neq 0 \\ x, & \text { if } y=0\end{cases}
$$

then $(H, \circ, 0)$ is a hyper BCK-algebra.

Proposition 3 (see [10]). In a hyper BCK-algebra $H$, the condition (HK3) is equivalent to the following condition: for all $x, y \in H, x \circ y \ll\{x\}$.

Proposition 4 (see [10]). In a hyper BCK-algebra $H$, the following hold.

(1) For all $x \in H, x \circ 0 \ll\{x\}, 0 \circ x \ll\{0\}$, and $0 \circ 0 \ll\{0\}$.

(2) For any nonempty subsets $A, B$ and $C \subseteq H,(A \circ B) \circ C=$ $(A \circ C) \circ B, A \circ B \ll A, 0 \circ A \ll\{0\}$.

Proposition 5 (see [7, 10]). In any hyper BCK-algebra $H$, the following properties hold: for all $x, y, z \in H$, and for any nonempty subsets $A, B \subseteq H$,

(1) $0 \circ 0=\{0\}$,

(2) $0 \ll x$,

(3) $x \ll x$,

(4) $A \ll A$,

(5) $A \subseteq B \Rightarrow A \ll B$,
(6) $0 \circ x=\{0\}$,

(7) $0 \circ A=\{0\}$,

(8) $A \ll\{0\} \Rightarrow A=\{0\}$,

(9) $A \circ B \ll A$,

(10) $x \in x \circ 0$,

(11) $x \circ 0 \ll\{y\} \Rightarrow x \ll y$,

(12) $y \ll z \Rightarrow x \circ z \ll x \circ y$,

(13) $x \circ y=\{0\} \Rightarrow(x \circ z) \circ(y \circ z)=\{0\}, x \circ z \ll y \circ z$,

(14) $A \circ\{0\}=\{0\} \Rightarrow A=\{0\}$,

(15) $x \circ 0=\{x\}$,

(16) $x \in x \circ(x \circ x)$,

(17) $0 \circ(x \circ 0)=\{0\}$ and $x \circ(0 \circ x)=\{x\}$.

Definition 6 (see [10]). Let $I$ be a nonempty subset of a hyper BCK-algebra $H$. Then $I$ is said to be a hyper BCK-ideal of $H$ if

(1) $0 \in I$,

(2) $x \circ y \ll I$ and $y \in I$ imply $x \in I$ for all $x, y \in H$.

Note that if $I$ is a hyper BCK-ideal of hyper BCK-algebra $H$, then $x \in I$ implies $x \circ y \subseteq I$.

Definition 7 (see [19]). A hyper BCK-algebra is called bounded if there is an element $e \in H$ such that $x \ll e$ for all $x \in H$. This element $e$ is called the unit of $H$, and we denote a bounded hyper BCK-algebra $(H ; \circ, 0, e)$ simply by $H$.

Example 8. Let $H=\{0,1,2\}$. Define a hyperoperation “。” on $H$ as follows:

\begin{tabular}{c|ccc}
$\circ$ & 0 & 1 & 2 \\
\hline 0 & $\{0\}$ & $\{0\}$ & $\{0\}$ \\
1 & $\{1\}$ & $\{0,1\}$ & $\{0,1\}$ \\
2 & $\{2\}$ & $\{1,2\}$ & $\{0,1,2\} ;$
\end{tabular}

then $H$ is a bounded hyper BCK-algebra, where $0 \ll 1 \ll 2$.

In a hyper BCK-algebra $(H, \circ, 0)$, we define a hyperoperation " $\wedge$ " by $x \wedge y=y \circ(y \circ x)$ for all $x, y \in H$. For all $A, B \subseteq H$, $A \wedge B=\bigcup_{a \in A, b \in B} a \wedge b$. In general, $x \wedge y \neq y \wedge x$.

Proposition 9. Let $H$ be a hyper BCK-algebra. Then for any $x, y, z \in H$,

(1) $x \wedge y \ll\{y\}$,

(2) $y \ll x \Rightarrow x \wedge y=\{y\}$,

(3) $y \ll z \Rightarrow y \wedge x \ll z \wedge x$

Proof.

(1) By (HK3) of Definition 1, we have $x \wedge y=y \circ(y \circ$ $x) \subseteq y \circ H \ll\{y\}$. Therefore $x \wedge y \ll\{y\}$.

(2) Suppose that $y \ll x$; then $0 \in y \circ x, y \circ 0 \subseteq y \circ(y \circ x)$. So $y \in y \circ 0$ by Proposition 5 (10). Hence $y \in y \circ(y \circ x)$ which implies $\{y\} \subseteq x \wedge y$; that is, $\{y\} \ll x \wedge y$. Also $x \wedge y=\{y\}$ by (1). 
(3) By Proposition 5 (12), $y \ll z \Rightarrow x \circ z \ll x \circ y \Rightarrow$ $x \circ(x \circ y) \ll x \circ(x \circ z)$; that is, $y \wedge x \ll z \wedge x$.

Let $(H, \circ, 0, e)$ be a bounded hyper BCK-algebra. Then we define $x^{-}:=e \circ x$ for any $x \in H$.

Proposition 10. Let $(H, \circ, 0, e)$ be a bounded hyper BCKalgebra; the following hold:

(1) $x \ll y \Rightarrow y^{-} \ll x^{-}$,

(2) $x^{-} \circ y=y^{-} \circ x$,

(3) $\left(x^{-} \circ y\right) \circ z=\left(x^{-} \circ z\right) \circ y=\left(z^{-} \circ x\right) \circ y=\left(z^{-} \circ y\right) \circ x=$ $\left(y^{-} \circ z\right) \circ x=\left(y^{-} \circ x\right) \circ z$.

Proof. (1) Assume that $x \ll y$; it is clear $y^{-} \ll x^{-}$by Proposition 5.

(2) By (HK2), $x^{-} \circ y=(e \circ x) \circ y=(e \circ y) \circ x=y^{-} \circ x$.

(3) By (2) and (HK2), it is easy to prove (3).

An MV-algebra is an algebra $\left(A, \oplus,{ }^{-}, 0\right)$ of type $(2,1,0)$ such that $(1) \oplus$ is commutative and associative, (2) $x \oplus 0=x$, (3) $x \oplus 0^{-}=0^{-}$, (4) $x^{--}=x$, and (5) $y \oplus\left(y \oplus x^{-}\right)^{-}=x \oplus$ $\left(x \oplus y^{-}\right)^{-}$. In [20], we know that MV-algebras are categorically equivalent to bounded commutative BCK-algebras. Now we discuss the relation between a bounded hyper BCK-algebra and an MV-algebra.

Define $S(H)=\{x \in H: x \circ x=\{0\}\}$. Then we get the following results.

Lemma 11 (see [6]). Every hyper BCK-algebra $H$ is a BCKalgebra if and only if $H=S(H)$.

Theorem 12. Let $(H, \circ)$ be a hyper BCK-algebra. If $H$ satisfies the condition $x \circ(x \circ y)=y \circ(y \circ x)$, for all $x, y \in H$, then

(1) $x \circ(x \circ 0)=\{0\}$, for all $x \in H$,

(2) $x \circ 0=\{x\}$, for all $x \in H$,

(3) $x \circ x=\{0\}$, for all $x \in H$,

(4) $H=S(H)$.

Proof. (1) Suppose that $x \circ(x \circ y)=y \circ(y \circ x)$, for all $x, y \in H$. Let $x \in H$; then we have $x \circ(x \circ 0)=0 \circ(0 \circ x)=\{0\}$ by Proposition 5, and so $x \circ(x \circ 0)=\{0\}$.

(2) Let $a \in x \circ 0$ for every $x \in H$. Since $x \circ 0 \ll\{x\}$, we get $a \ll x$. On the other hand, $x \circ a \subseteq x \circ(x \circ 0)=\{0\}$ and thus $x \circ a=\{0\}$. Hence $x \ll a$, and we conclude that $x=a$. Consequently $x \circ 0=\{x\}$.

(3) For all $x \in H$, we get $x \circ x=\{0\}$ by (1) and (2).

(4) By (3) and Lemma 11 we have $H=S(H)$.

From Theorem 12, we obtain the relation between hyper BCK-algebras and MV-algebras.

Corollary 13. Let $(H, \circ, 0, e)$ be a bounded hyper BCK-algebra with the condition $x \circ(x \circ y)=y \circ(y \circ x)$, for all $x, y \in H$. Then $(H, \circ, 0, e)$ is a bounded commutative BCK-algebra. We define $x^{-}=e \circ x$ for all $x \in H$ and $x \oplus y=\left(x^{-} \circ y\right)^{-}$for all $x, y \in H$. Then $\left(H, \oplus,{ }^{-}, 0, e\right)$ is an $M V$-algebra.
Now, let us review the structure of quotient hyper BCK-algebras on which we consider inf-Bosbach states in Section 4.

Definition 14 (see [19]). Let $\theta$ be an equivalence relation on a hyper BCK-algebra $H$ and $A, B \subseteq H$. Then,

(1) $A \theta B$ means that there exist $a \in A$ and $b \in B$ such that $a \theta b$

(2) $A \bar{\theta} B$ means that for all $a \in A$ there exists $b \in B$ such that $a \theta b$ and for all $b \in B$ there exists $a \in A$ such that $a \theta b$;

(3) $\theta$ is called a congruence relation on $H$; if $x \theta y$ and $x^{\prime} \theta y^{\prime}$ then $x \circ x^{\prime} \theta y \circ y^{\prime}$, for all $x, y, x^{\prime}, y^{\prime} \in H$;

(4) $\theta$ is called a regular relation on $H$; if $x \circ y \theta\{0\}$ and $y \circ x \theta\{0\}$, then $x \theta y$ for all $x, y \in H$.

Lemma 15 (see [19]). Let $\theta$ be an equivalence relation on $H$ and $A, B, C \subseteq H$. If $A \bar{\theta} B$ and $B \bar{\theta} C$, then $A \bar{\theta} C$.

Lemma 16 (see [19]). Let $\theta$ be an equivalence relation on $H$. Then the following statements are equivalent:

(1) $\theta$ is a congruence relation on $H$;

(2) if $x \theta y$, then $x \circ a \bar{\theta} y \circ a$ and $a \circ x \bar{\theta} a \circ y$, for all $a, x, y \in H$.

Theorem 17 (see [19]). Let $\theta$ and $\theta^{\prime}$ be two regular congruence relations on $H$ such that $[0]_{\theta}=[0]_{\theta^{\prime}}$. Then $\theta=\theta^{\prime}$.

Lemma 18 (see [19]). Let $\theta$ be a congruence relation on $H$. Then $[0]_{\theta}$ is a strong hyper BCK-ideal of $H$.

Theorem 19 (see [19]). Let $\theta$ be a regular congruence relation on $H, I=[0]_{\theta}$ and $H / I=\left\{I_{x}: x \in H\right\}$, where $I_{x}=[x]_{\theta}$ for all $x \in H$. Then $(H / I, \circ,<)$ is a hyper BCK-algebra, which is called a quotient hyper BCK-algebra, where "o" and " $<$ " are defined as follows: $I_{x} \circ I_{y}=\left\{I_{z}: z \in x \circ y\right\}$ and $I_{x}<I_{y} \Leftrightarrow I_{0} \in I_{x} \circ I_{y}$.

\section{States on Bounded Hyper BCK-Algebras}

In this section, the concepts of Bosbach states and infBosbach states on a bounded hyper BCK-algebra are defined, and its properties are studied.

In what follows in the paper, we denote a bounded hyper BCK-algebra by $(H, \circ, 0, e)$ or $H$, unless otherwise specified.

Definition 20. A function $s: P^{*}(H) \rightarrow[0,1]$ is called a Bosbach state on $H$ if it satisfies the following conditions:

(1) $s(0)=0, s(e)=1$,

(2) $s(x)+s(y \circ x)=s(y)+s(x \circ y)$, for any $x, y \in H$.

Example 21. Let $H$ be defined in Example 8. We define $s(0)=$ $0, s(1)=1 / 2, s(2)=1, s(\{0,1\})=0, s(\{0,2\})=0, s(\{1,2\})=$ $1 / 2$, and $s(\{0,1,2\})=0$. Then $s$ is a Bosbach state on $H$.

Definition 22. A function $s: H \rightarrow[0,1]$ is called an infBosbach state on $H$ if it satisfies the following conditions:

(1) $s(0)=0, s(e)=1$, 
(2) $s(x)+s(y \circ x)=s(y)+s(x \circ y)$, for any $x, y \in H$,

where $s(x)$ is an abbreviation of $s(\{x\})$, and $s(A)$ is defined by $s(A)=\inf \{s(t) \mid t \in A\}$ for any $A \subseteq H$.

Example 23. Let $H$ be defined in Example 8. Assume that $s$ is an inf-Bosbach state on $H$. Then we have $s(0)=0$ and $s(2)=$ 1 . Assume $s(1)=a$. Since $s(1)+s(2 \circ 1)=s(2)+s(1 \circ 2)$, we have $a+s(1)=1+s(0)$ and hence $a+a=1+0$. Therefore $a=1 / 2$. It follows that $s$ is the unique inf-Bosbach state on $H$.

The following example shows that not every bounded hyper BCK-algebra has an inf-Bosbach state.

Example 24. Let $H=\{0,1,2,3\}$. Define a hyperoperation “。” on $H$ as follows:

\begin{tabular}{c|cccc}
$\circ$ & 0 & 1 & 2 & 3 \\
\hline 0 & $\{0\}$ & $\{0\}$ & $\{0\}$ & $\{0\}$ \\
1 & $\{1\}$ & $\{0\}$ & $\{0\}$ & $\{0\}$ \\
2 & $\{2\}$ & $\{1\}$ & $\{0,1\}$ & $\{1\}$ \\
3 & $\{3\}$ & $\{1\}$ & $\{0\}$ & $\{0,1\}$.
\end{tabular}

Then $H$ is a bounded hyper BCK-algebra, where $0 \ll 1 \ll$ $3 \ll 2$. Let $s(0)=0, s(1)=a, s(3)=b$, and $s(2)=1$. From $s(x)+s(y \circ x)=s(y)+s(x \circ y)$, taking $x=1, y=2$, we get $a=1 / 2$. Taking $x=1, y=3$, we get $b=1$. Taking $x=2$, $y=3$, we get $1=b+a=1+1 / 2$. It is a contradiction. Hence, $H$ does not admit any inf-Bosbach state.

Lemma 25. Let $s$ be an inf-Bosbach state on $H$. Then $s$ is a Bosbach state on $H$.

Then we give some basic properties of inf-Bosbach states on hyper BCK-algebras.

Proposition 26. Let s be an inf-Bosbach state on $H$. Then the following hold:

(1) $x \ll y \Rightarrow s(y \circ x)=s(y)-s(x)$,

(2) $x \ll y \Rightarrow s(x) \leq s(y)$,

(3) $s(y \circ(y \circ(y \circ x)))=s(y \circ x)$.

Proof. (1) and (2) are trivial. By Proposition 3 and (1), we get that $s(y \circ(y \circ(y \circ x)))=s(y)-s(y \circ(y \circ x))=s(y)-(s(y)-$ $s(y \circ x))=s(y \circ x)$. So (3) holds.

Proposition 27. Let $s$ be an inf-Bosbach state on $H$. Then,

(1) $s(x \wedge y)=s(y)-s(y \circ x)$

(2) $s(x \wedge y)=s(y \wedge x)$,

(3) $s\left(x^{-}\right)=1-s(x), s\left(x^{--}\right)=s(x)$,

(4) $s\left(x^{-} \circ y\right)=s\left(y^{-} \circ x\right), s\left(x \circ y^{-}\right)=s\left(y \circ x^{-}\right)$,

(5) $y \ll x \Rightarrow s(x \circ y)=s\left(y^{-} \circ x^{-}\right)$.

Proof. (1) Note that $y \circ x \ll\{y\}$ by Proposition 3, so we have $s(x \wedge y)=s(y \circ(y \circ x))=s(y)-s(y \circ x)$.

(2) Combining (1) and Definition 22, we get $s(x \wedge y)-$ $s(y \wedge x)=(s(y)-s(y \circ x))-(s(x)-s(x \circ y))=s(y)+s(x \circ$ $y)-(s(x)+s(y \circ x))=0$. Thus $s(x \wedge y)=s(y \wedge x)$.
(3) Since $x \ll e$, then $s\left(x^{-}\right)=s(e \circ x)=s(e)-s(x)=$ $1-s(x)$. Moreover $s\left(x^{--}\right)=1-s\left(x^{-}\right)=1-(1-s(x))=s(x)$.

(4) By Proposition 10, we get $x^{-} \circ y=y^{-} \circ x$. So $s\left(x^{-} \circ y\right)=$ $s\left(y^{-} \circ x\right)$ and $s\left(x \circ y^{-}\right)=s(x)+s\left(y^{-} \circ x\right)-s\left(y^{-}\right)=s(x)+$ $s\left(x^{-} \circ y\right)-1+s(y)=s(y)+s\left(x^{-} \circ y\right)-s\left(x^{-}\right)=s\left(y \circ x^{-}\right)$.

(5) Suppose $y \ll x$; then we have $x^{-} \ll y^{-}$. So $s(x \circ y)=$ $s(x)-s(y)=(1-s(y))-(1-s(x))=s\left(y^{-}\right)-s\left(x^{-}\right)=s\left(y^{-}\right.$。 $\left.x^{-}\right)$.

The following theorem gives an equivalent characterization of inf-Bosbach states.

Theorem 28. Let $s: H \rightarrow[0,1]$ satisfy $s(e)=1$. Then the following are equivalent:

(1) $s$ is an inf-Bosbach state on $H$;

(2) $s(x \wedge y)=s(y \wedge x)$ and $x \ll y \Rightarrow s(y \circ x)=s(y)-s(x)$.

Proof. (1) $\Rightarrow$ (2) It follows from Proposition 27 (2) and Proposition 26 (1)

$(2) \Rightarrow(1) s(0 \circ 0)=s(0)=s(0)-s(0)=0$. Since $s(x \wedge y)=$ $s(y \wedge x)$, we obtain $s(y)-s(y \circ x)=s(x)-s(x \circ y)$; that is, $s(x)+s(y \circ x)=s(y)+s(y \circ x)$.

Theorem 29. Let $s$ be an inf-Bosbach state on $H$. Define $K=$ $\operatorname{Ker}(s)=\{a \in H \mid s(a)=0\}$ which is called the kernel of the inf-Bosbach state s. Then $K$ is a hyper BCK-ideal of $H$.

Proof. Clearly, $0 \in K$. Let $x \circ y \ll K$ and $y \in K$. So $s(y)=0$. Since $x \circ y \ll K$, then for all $t \in x \circ y$, there is $i \in K$ such that $t \ll i$. Since $s$ is order-preserving, we have $s(t) \leq s(i)=0$. Hence $s(t)=0$; that is, $s(x \circ y)=0$. Also note that $y \circ x \ll\{y\}$, so $s(y \circ x) \leq s(y)=0$. This shows that $s(y \circ x)=0$. We obtain $s(x)=0$ by the definition of inf-Bosbach state $s$. Therefore, we have $x \in K$.

\section{States on Quotient Hyper BCK-Algebras}

In this section, we study the inf-Bosbach states on quotient hyper BCK-algebras.

Definition 30. Let $s$ be an inf-Bosbach state and let $\theta$ be a congruence relation on $(H, \circ, 0, e)$. Then $s$ is called $\theta$-compatibled $s(x)=s(y)$ if and only if $x \theta y$ for all $x, y \in H$.

Lemma 31. Let $\theta$ be a regular congruence relation and let $s$ be $a \theta$-compatibled inf-Bosbach state on $H$. Define $I=[0]_{\theta}$. Then in the bounded quotient hyper BCK-algebra $\left(H / I, \circ, I, I_{e}\right)$, where $I_{x}=[x]_{\theta}, I=I_{0}$ and $I_{x \circ y}=I_{x} \circ I_{y}$, the following hold:

(1) $I_{x}<I_{y}$ if and only if $s(x \circ y)=0$,

(2) $I_{x}=I_{y}$ if and only if $s(x)=s(y)$.

Proof. (1) Since $I_{x}<I_{y}$ implies $I \in I_{x} \circ I_{y}$, then there exists $z \in x \circ y$ such that $z \theta 0$. By Definition 30, we get that $s(z)=0$. Then $s(x \circ y)=\inf \{s(t): t \in x \circ y\}=0$. On the other hand, suppose that $s(x \circ y)=0$. Then there is $z \in x \circ y$ such that $z \theta 0$. Moreover we get $I_{z}=I_{0}=I$; that is, there is $I_{z} \in I_{x} \circ I_{y}$ such that $I_{z}=I$. This means that $I_{x}<I_{y}$. 
(2) It is clear that $I_{x}=I_{y}$ if and only if $x \theta y$ if and only if $s(x)=s(y)$.

Theorem 32. Let $\theta$ be a regular congruence relation and let $s$ be a $\theta$-compatibled inf-Bosbach state on $H$. Take $I=[0]_{\theta}$. Define a map $\widehat{s}: H / I \rightarrow[0,1]$ by $\widehat{s}\left(I_{x}\right)=s(x)$ and $\widehat{s}\left(\left\{I_{x}: x \in\right.\right.$ $A\})=\inf \left\{\widehat{s}\left(I_{t}\right): t \in A\right\}$, for any $x \in H$ and $A \subseteq H$. Then $\widehat{s}$ is an inf-Bosbach state on $H / I$.

Proof. By Lemma 31, the definition of $\widehat{s}$ is well defined. Clearly, $\widehat{s}(I)=s(0)=0$ and $\widehat{s}\left(I_{e}\right)=s(e)=1$. Since $\widehat{s}\left(I_{x} \circ I_{y}\right)=\inf \left\{\widehat{s}\left(I_{t}\right): t \in x \circ y\right\}=\inf \{s(t): t \in x \circ y\}=s(x \circ y)$, then $\widehat{s}\left(I_{x}\right)+\widehat{s}\left(I_{y} \circ I_{x}\right)=s(x)+s(y \circ x)=s(y)+s(x \circ y)=$ $\widehat{s}\left(I_{y}\right)+\widehat{s}\left(I_{x}+I_{y}\right)$. Therefore, $\widehat{s}$ is an inf-Bosbach state on $H / I$.

Definition 33. Let $\theta$ be a regular congruence relation on $H$. Then $\theta$ is called o-compatibled if there is $t \in H$ such that $x \circ$ $y \subseteq[t]_{\theta}$ for all $x, y \in H$.

Lemma 34. Let $\theta$ be a o-compatibled regular congruence relation on $H$. Then there is $u \in H$ such that $x \wedge y \subseteq[u]_{\theta}$ for all $x, y \in H$.

Proof. Since $\theta$ is o-compatibled, so there exists $t \in H$ such that $y \circ x \subseteq[t]_{\theta}$ for all $x, y \in H$. Hence $x \wedge y=y \circ(y \circ$ $x) \subseteq y \circ[t]_{\theta}$. For any $a, b \in[t]_{\theta}$, we have $a \theta b$. Since $\theta$ is a congruence relation, then $y \circ a \bar{\theta} y \circ b$ by Lemma 16. Since $\theta$ is o-compatibled, there exists $u \in H$ such that $y \circ a \subseteq[u]_{\theta}$ and $y \circ b \subseteq[u]_{\theta}$. This shows that for any $w \in[t]_{\theta}, y \circ w$ is contained in the same equivalent class. Hence $y \circ[t]_{\theta} \subseteq[u]_{\theta}$. It follows that $x \wedge y \subseteq[u]_{\theta}$.

Lemma 35. Let $\theta$ be a o-compatibled regular congruence relation on $H$. Then $(x \wedge y) \bar{\theta}(y \wedge x)$ for all $x, y \in H$.

Proof. By Lemma 34, there exists $u \in H$ such that $x \wedge y \subseteq[u]_{\theta}$ for all $x, y \in H$. Similarly, there exists $v \in H$ such that $y \wedge x \subseteq$ $[v]_{\theta}$. By Proposition 27, $s(x \wedge y)=s(y \wedge x)$, and so $s(a)=s(b)$ for some $a \in x \wedge y$ and $b \in y \wedge x$. Since $s$ is $\theta$-compatibled, then $a \theta b$. Hence $[u]_{\theta}=[a]_{\theta}=[b]_{\theta}=[v]_{\theta}$. Therefore, $x \wedge y \subseteq[u]_{\theta}$ and $y \wedge x \subseteq[u]_{\theta}$, which implies $(x \wedge y) \bar{\theta}(y \wedge x)$.

Lemma 36. Let $\theta$ be a regular congruence relation on $H$ and $I=[0]_{\theta}$. Then for any $x, y \in H, I_{x} \wedge I_{y}=I_{x \wedge y}$ in $\left(H / I, \circ, I, I_{e}\right)$.

Proof. Note that $I_{x} \wedge I_{y}=I_{y} \circ\left(I_{y} \circ I_{x}\right)=I_{y} \circ\left\{I_{t}: t \in y \circ x\right\}=$ $\left\{I_{y} \circ I_{t}: t \in y \circ x\right\}=\left\{I_{u}: u \in y \circ t, t \in y \circ x\right\}=\left\{I_{u}: u \in\right.$ $y \circ(y \circ x)\}=\left\{I_{u}: u \in x \wedge y\right\}=I_{x \wedge y}$.

Lemma 37. Let $\theta$ be a o-compatibled regular congruence relation on $H$ and let $s$ be a $\theta$-compatibled inf-Bosbach state on $(H, \circ, 0, e)$. Then the bounded quotient hyper BCK-algebra $H / I$ is a bounded commutative BCK-algebra.

Proof. Note that $I_{x} \circ I_{y}=\left\{I_{z}, z \in x \circ y\right\}$. Since $\theta$ is o-compatibled, then there is $t \in H$ such that $x \circ y \subseteq[t]_{\theta}$. This shows that $\left|I_{x} \circ I_{y}\right|=1$ for all $x, y \in H$. It follows that $H / I$ is a BCK-algebra. Since $\theta$ is o-compatibled, then by Lemma 34, there is $u \in H$ such that $x \wedge y \subseteq[u]_{\theta}$. Hence
$I_{x} \wedge I_{y}=I_{x \wedge y} \subseteq I_{[u]_{\theta}}=I_{u}$. Note that $H / I$ is a BCK-algebra and by Lemma 36, we get $I_{x} \wedge I_{y}=I_{u}$. By Lemma 35, $I_{y} \wedge I_{x}=I_{u}$. Therefore, $I_{x} \wedge I_{y}=I_{y} \wedge I_{x}$.

Summarizing the above conclusions, we get the following result.

Theorem 38. Let $\theta$ be a o-compatibled regular congruence relation and let $s$ be a $\theta$-compatibled inf-Bosbach state on $H$. Take $I=[0]_{\theta}$. Define $I_{x} \oplus I_{y}=\left(\left(I_{x}\right)^{-} \circ I_{y}\right)^{-}$for all $I_{x}, I_{y} \in H / I$. Then $\left(H / I, \oplus^{-}\right)$is an MV-algebra. Moreover, the map $\widehat{s}: H / I \rightarrow[0,1]$ defined as Theorem 32 is an inf-Bosbach state on $H / I$ and the following hold:
(1) $\widehat{s}\left(I_{x} \circ I_{y}\right)=s(x \circ y)$,
(2) $\widehat{s}\left(I_{x}^{-}\right)=1-s(x)$,
(3) $\widehat{s}\left(I_{x} \oplus I_{y}\right)=1-s\left(x^{-} \circ y\right)$,
(4) $\widehat{s}\left(I_{x} \wedge I_{y}\right)=\widehat{s}\left(I_{y} \wedge I_{x}\right)=s(x \wedge y)=s(y \wedge x)$.

\section{Hyper Measures on Hyper BCK-Algebras}

In this section, we study the hyper measures on hyper BCKalgebras.

Define “*” on the real interval $X=[0, \infty)$ as follows: $x *$ $y=\max \{0, x-y\}$, for all $x, y \in X$. Then $(X, *, 0)$ is a BCKalgebra.

Definition 39. Let $(H, \circ, 0)$ be a hyper BCK-algebra. A map $m: P^{*}(H) \rightarrow[0, \infty)$ such that, for all $x, y \in H$,

(1) $m(x \circ y)=m(x)-m(y)$ wherever $y \ll x$ is said to be a hyper measure;

(2) if $H$ is bounded, $e$ is the unit of $H$, and $m$ is a hyper measure with $m(e)=1$, then $m$ is said to be a hyper state;

(3) $m(x \circ y)=m(x) * m(y)$ is said to be a hyper measuremorphism;

(4) if $H$ is bounded, $e$ is the unit of $H$, and $m$ is a hyper measure-morphism with $m(e)=1$, then $m$ is said to be a hyper state-morphism.

Obviously any hyper measure-morphism on a hyper BCK-algebra $H$ is a hyper measure.

Proposition 40. Let $m$ be a hyper measure on hyper BCKalgebra $(H, \circ, 0)$. Then for all $x, y \in H$, one has the following:

(1) $m(0)=0$,

(2) $x \ll y$ implies $m(x) \leq m(y)$,

(3) $x \ll y$ implies $m(x \wedge y)=m(x)$,

(4) $m(x \circ(y \wedge x))=m(x \circ y)$.

Proof. (1) Clearly we have $m(0)=m(0 \circ 0)=m(0)-m(0)=0$.

(2) Since $x \ll y$ implies $m(y \circ x)=m(y)-m(x) \geq 0$, then $m(x) \leq m(y)$.

(3) Note that $x \ll y$ implies $m(x \wedge y)=m(y \circ(y \circ x))=$ $m(y)-m(y \circ x)=m(y)-(m(y)-m(x))=m(x)$. 
(4) By Definition 39, $m(x \circ(y \wedge x))=m(x \circ(x \circ(x \circ y)))=$ $m(x)-m(x \circ(x \circ y))=m(x)-(m(x)-m(x \circ y))=m(x \circ y)$.

Theorem 41. Let $m$ be a hyper state-morphism on $(H, \circ, 0, e)$. Then $m$ is a Bosbach state on $H$.

Proof. Clearly, $m(0)=0$ and $m(e)=1$. By Proposition 40, we have $m(x) \in[0,1]$ for all $x \in H$. Moreover, $m(x)+m(y \circ$ $x)=m(x)+\max \{0, m(y)-m(x)\}=\max \{m(x), m(y)\}=$ $\max \{m(x)-m(y), 0\}+m(y)=m(x \circ y)+m(y)$. Therefore $m$ is a Bosbach state on $H$.

Proposition 42. Let $m$ be a hyper state-morphism on $(H, \circ, 0, e)$. Then for all $x, y \in H$,

(1) $m(x \wedge y)=m(y)-m(y \circ x)$,

(2) $m(x \wedge y)=m(y \wedge x)$,

(3) $m\left(x^{-}\right)=1-m(x), m\left(x^{--}\right)=m(x)$,

(4) $m\left(x^{-} \circ y\right)=m\left(y^{-} \circ x\right)$.

Proof. (1) Since $x \circ y \ll x$, then $m(y \wedge x)=m(x \circ(x \circ y))=$ $m(x)-m(x \circ y)$.

(2) Observe that $m(x)+m(y \circ x)=m(y)+m(x \circ y)$ by Theorem 41. So, we obtain $m(x \wedge y)=m(y \wedge x)$.

(3) Since $x \ll e$, then $m\left(x^{-}\right)=m(e \circ x)=m(e)-m(x)=$ $1-m(x)$ and $m\left(x^{--}\right)=1-m\left(x^{-}\right)=m(x)$.

(4) Since $x^{-} \circ y=y^{-} \circ x$, then $m\left(x^{-} \circ y\right)=m\left(y^{-} \circ x\right)$.

Definition 43 (see [10]). A hyper BCK-ideal I of a hyper BCKalgebra $H$ is said to be reflexive if $x \circ x \subseteq I$ for all $x \in H$.

Theorem 44 (see [19]). Let I be a reflexive hyper BCK-ideal of a hyper BCK-algebra $H$. A relation $\Theta$ on $H$ is defined as follows: for all $x, y \in H$,

$$
x \Theta y \Longleftrightarrow x \circ y \subseteq I, \quad y \circ x \subseteq I .
$$

Then $\Theta$ is a regular congruence relation on $H$ and $I=[0]_{\Theta}$. Moreover, $H / I$ is a BCK-algebra.

Lemma 45. Let $m$ be a hyper state-morphism on $H$. Then $\operatorname{Ker}(m)=\{x \in H: m(x)=0\}$ is a reflexive hyper BCK-ideal.

Proof. Clearly, $0 \in \operatorname{Ker}(m)$. Assume that $x \circ y \ll \operatorname{Ker}(m)$ and $y \in \operatorname{Ker}(m)$. For all $t \in x \circ y$, there exists $u \in \operatorname{Ker}(m)$, such that $t \ll u$. By Proposition 40, we have $m(t) \leq m(u)=0$. Hence $m(x \circ y)=0$. Since $y \circ x \ll\{y\}$, then for all $t \in y \circ x$, such that $t \ll y$. By Proposition $40, m(t) \leq m(y)=0$. Hence $m(y \circ x)=0$. By Theorem $41, m$ is a Bosbach state, and so we have $m(x)+m(y \circ x)=m(y)+m(x \circ y)$. Thus $m(x)=0$. Therefore, $\operatorname{Ker}(m)$ is a hyper BCK-ideal. Since $m(x \circ x)=$ $\max \{0, m(x)-m(x)\}=0$, then $x \circ x \subseteq \operatorname{Ker}(m)$. So, $\operatorname{Ker}(m)$ is a reflexive hyper BCK-ideal.

Theorem 46. Let $m$ be a hyper state-morphism on $H$. A relation $\Theta$ on $H$ is defined as follows:

$$
x \Theta y \Longleftrightarrow x \circ y \subseteq \operatorname{Ker}(m), \quad y \circ x \subseteq \operatorname{Ker}(m)
$$

Then $(H / \operatorname{Ker}(m), \circ, \overline{0}, \bar{e})$ is a bounded commutative BCKalgebra, where $\bar{x}=x / \operatorname{Ker}(m)$ and $\bar{x} \circ \bar{y}=(x \circ y) / \operatorname{Ker}(m)$ for all $\bar{x}, \bar{y} \in H / \operatorname{Ker}(m)$. And $\bar{x} \leq \bar{y}$ is defined by $\bar{x} \circ \bar{y}=\overline{0}$.

Moreover, define a map $M: H / \operatorname{Ker}(m) \rightarrow[0,1]$ by $M(\bar{x})=m(x), \bar{x} \in H / \operatorname{Ker}(m)$. Then,

(1) $\bar{x} \leq \bar{y}$ if and only if $m(x \circ y)=0$ if and only if $M(\bar{x}) \leq$ $M(\bar{y})$;

(2) $\bar{x}=\bar{y}$ if and only if $m(x \circ y)=m(y \circ x)=0$ if and only if $M(\bar{x})=M(\bar{y})$;

(3) $M$ is a state-morphism on $\mathrm{H} / \operatorname{Ker}(m)$.

Proof. By Proposition 42, $m(x \wedge y)=m(y \wedge x)$. Then for all $t \in x \wedge y$ there exists $s \in y \wedge x$ such that $m(t)=m(s)$ and for all $t^{\prime} \in y \wedge x$ there exists $s^{\prime} \in x \wedge y$ such that $m\left(t^{\prime}\right)=m\left(s^{\prime}\right)$. Therefore, $m(t \circ s)=m(s \circ t)=m\left(t^{\prime} \circ s^{\prime}\right)=$ $m\left(s^{\prime} \circ t^{\prime}\right)=0$, which implies $t \circ s, s \circ t, t^{\prime} \circ s^{\prime}, s^{\prime} \circ t^{\prime} \subseteq$ $\operatorname{Ker}(m)$. So $t \Theta$ s (i.e., $t / \operatorname{Ker}(m)=s / \operatorname{Ker}(m))$ and $t^{\prime} \Theta s^{\prime}$ (i.e., $\left.t^{\prime} / \operatorname{Ker}(m)=s^{\prime} / \operatorname{Ker}(m)\right)$. In $H / \operatorname{Ker}(m), \bar{x} \wedge \bar{y}=\bar{y} \circ(\bar{y} \circ \bar{x})=$ $\bar{y} \circ((y \circ x) / \operatorname{Ker}(m))=(y \circ(y \circ x)) / \operatorname{Ker}(m)$. So, we have $\bar{x} \circ(\bar{x} \circ \bar{y})=\bar{y} \circ(\bar{y} \circ \bar{x})$. By Theorem $44, H / \operatorname{Ker}(m)$ is a BCKalgebra. Combining the above arguments, we get $H / \operatorname{Ker}(m)$ is a bounded commutative BCK-algebra.

In the following, we prove the second part of the theorem.

(1) Note that $\bar{x} \leq \bar{y}$ if and only if $\bar{x} \circ \bar{y}=\overline{0}$ if and only if $(x \circ y) / \operatorname{Ker}(m)=0 / \operatorname{Ker}(m)$, which implies for all $t \in x \circ y$ we have $t \Theta 0$. Then we get $t \circ 0=\{t\} \subseteq$ $\operatorname{Ker}(m)$, which implies $m(t)=0$. Thus $m(x \circ y)=$ 0 . By $m(x \circ y)=\max \{0, m(x)-m(y)\}$, we have $m(x) \leq m(y)$; that is, $M(\bar{x}) \leq M(\bar{y})$. Conversely, suppose that $M(\bar{x}) \leq M(\bar{y})$; we have $m(x) \leq m(y)$. It follows from the definition of hyper state-morphisms that we have $m(x \circ y)=0$. This means that for all $t \in x \circ y$ we have $t / \operatorname{Ker}(m)=0 / \operatorname{Ker}(m)$, which implies $(x \circ y) / \operatorname{Ker}(m)=\bar{x} \circ \bar{y}=0 / \operatorname{Ker}(m)$, or $\bar{x} \circ \bar{y}=\overline{0}$. That is, $\bar{x} \leq \bar{y}$.

(2) Similar to (1), we can prove (2).

(3) By (2), for all $\bar{x}, \bar{y} \in H / \operatorname{Ker}(m), \bar{x}=\bar{y}$ if and only if $m(x)=m(y)$ if and only if $M(\bar{x})=M(\bar{y})$. Therefore, the definition of $M$ is well defined. It is obvious that $M(\overline{0})=m(0)=0$ and $M(\bar{e})=m(e)=1$. Note that $M(\bar{x} \circ \bar{y})=M((x / \operatorname{Ker}(m)) \circ(y / \operatorname{Ker}(m)))=M((x \circ$ $y) / \operatorname{Ker}(m))=m(x \circ y)=\max \{0, m(x)-m(y)\}=$ $\max \{0, M(\bar{x})-M(\bar{y})\}=M(\bar{x}) * M(\bar{y})$. Therefore, $M$ is a state-morphism on $H / \operatorname{Ker}(m)$.

Corollary 47. In $(H / \operatorname{Ker}(m), \circ, \overline{0}, \bar{e})$ as Theorem 46, define $\bar{x}=\bar{e} \circ \bar{x}$, and $\bar{x} \oplus \bar{y}=\left(\bar{x}^{-} \circ \bar{y}\right)^{-}$for all $\bar{x}, \bar{y} \epsilon$ $H / \operatorname{Ker}(m)$. Then $\left(H / \operatorname{Ker}(m), \oplus,{ }^{-}\right)$is an $M V$-algebra. The map $M: H / \operatorname{Ker}(m) \rightarrow[0,1]$ defined as Theorem 46 is a statemorphism on $M V$-algebra $H / \operatorname{Ker}(m)$, and

(1) $M(\bar{x} \circ \bar{y})=m(x \circ y)$,

(2) $M\left(\bar{x}^{-}\right)=1-m(x)$,

(3) $M(\bar{x} \oplus \bar{y})=1-m\left(x^{-} \circ y\right)$,

(4) $M(\bar{x} \wedge \bar{y})=M(\bar{y} \wedge \bar{x})=m(x \wedge y)=m(y \wedge x)$. 


\section{Conclusions}

In this paper, we mainly study the state theory on hyper structures and introduce a notion of states on hyper BCKalgebras. In order to adapt a state to hyper operation, we define the state on a subset by $s(A)=\inf \{s(t): t \in A\}$. Using the definitions of o-compatibled regular congruence relations and $\theta$-compatibled inf-Bosbach states on hyper $\mathrm{BCK}$-algebras, we prove that the quotient structure of a bounded hyper BCK-algebra is an MV-algebra. Moreover, we define hyper measures on hyper BCK-algebras; then we introduce hyper states, hyper measure-morphisms, and hyper state-morphisms. We prove that a hyper state-morphism on hyper BCK-algebra is a Bosbach state. In the further work, we will solve the problem of how to define a state on a bounded hyper BCK-algebra to make the quotient structure form a hyper MV-algebra.

\section{Conflict of Interests}

The authors declare that there is no conflict of interests regarding the publication of this paper.

\section{Acknowledgments}

The authors are extremely grateful to the editor and the referees for their valuable comments and helpful suggestions which help to improve the presentation of this paper. This research is partially supported by a Grant from Graduate Higher Achievement Foundation of Northwest University (YC13055) and Graduate Independent Innovation Foundation of Northwest University (YZZ12061).

\section{References}

[1] F. Marty, "Sur une generalization de la notion de groupe," in Proceedings of the 8th International Congress on Industrial and Applied Mathematics, pp. 45-49, Scandinaves, Stockholm, Sweden, 1934.

[2] P. Corsini, Prolegomena of Hypergroup Theory, Aviani editore, 2nd edition, 1993.

[3] P. Corsini, "Feebly canonical and 1-hypergroups, Acta Universitatis Carolinae," Mathematica et Physica, vol. 24, no. 2, pp. 4956, 1983.

[4] M. Krasner, "A class of hyperrings and hyperfileds," International Journal of Applied Mathematics, vol. 2, pp. 307-312, 1983.

[5] G. G. Massouros, "Hypercompositional structures from the computer theory," Ratio Mathematica, vol. 13, pp. 37-42, 1999.

[6] Y. B. Jun, M. M. Zahedi, X. L. Xin, and R. A. Borzooei, "On hyper BCK-algebras," Italian Journal of Pure and Applied Mathematics, vol. 8, pp. 127-136, 2000.

[7] G. R. Biyogmam, O. A. Heubo-Kwegna, and J. B. Nganou, "Super implicative hyper BCK-algebras," International Journal of Pure and Applied Mathematics, vol. 76, no. 2, pp. 267-275, 2012.

[8] X. L. Xin, "Hyper BCI-algebras," Dscussiones Mathematicae General Algebra and Applications, vol. 26, pp. 5-19, 2006.

[9] J. Zhan, D. Xiang, and Z. Tan, "Fuzzy multiply positive implicative hyper BCK-ideals of hyper BCK-algebras," International Journal of Mathematics and Mathematical Sciences, vol. 2004, no. 24, pp. 1239-1253, 2004.
[10] Y. B. Jun and X. L. Xin, "Positive impliacative Hyper BCK-algebras," Scientiae Mathematicae Japonicae, vol. 55, pp. 97-106, 2002.

[11] Y. B. Jun, M. S. Kang, and H. S. Kim, "Bipolar fuzzy hyper BCKideals in hyper BCK-algebras," Iranian Journal of Fuzzy Systems, vol. 8, no. 2, pp. 105-120, 2011.

[12] C. C. Chang, "Algebraic analysis of many valued logics," Transactions of the American Mathematical Society, vol. 88, no. 2, pp. 467-490, 1958.

[13] D. Mundici, "Averaging the truth-value in Łukasiewicz logic," Studia Logica, vol. 55, no. 1, pp. 113-127, 1995.

[14] L. C. Ciungu, A. Dvurečenskij, and M. Hyčko, "State BL-algebras," Soft Computing, vol. 15, no. 4, pp. 619-634, 2011.

[15] L. Z. Liu, "On the existence of states on MTL-algebras," Information Sciences, vol. 220, pp. 559-567, 2013.

[16] A. Dvurečenskij, "Measures and states on BCK-algebras," Atti del Seminario Matematico e Fisico Dell'universitã di Modena, vol. 47, pp. 511-528, 1996.

[17] L. Corina Ciungu and A. Dvurečenskij, "Measures, states and de Finetti maps on pseudo-BCK algebras," Fuzzy Sets and Systems, vol. 161, no. 22, pp. 2870-2876, 2010.

[18] A. Dvurečenskij and S. Pulmannová, New Trends in Quantum Structures, Kluwer Academic, Ister Science, Bratislava, Slovakia, 2000.

[19] R. A. Borzooei and H. Harizavi, "Regular congruence relations on hyper BCK-algebras," Scientiae Mathematicae Japonicae, vol. 61, no. 1, pp. 83-98, 2005.

[20] D. Mundici, "MV-algebras are categorically equivalent to bounded commutative BCK-algebras," Mathematica Japonica, vol. 31, no. 6 , pp. 889-894, 1986. 


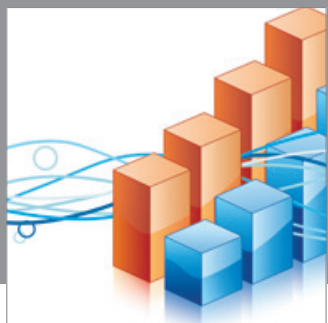

Advances in

Operations Research

mansans

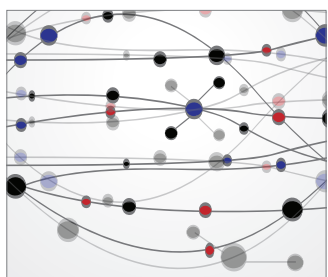

The Scientific World Journal
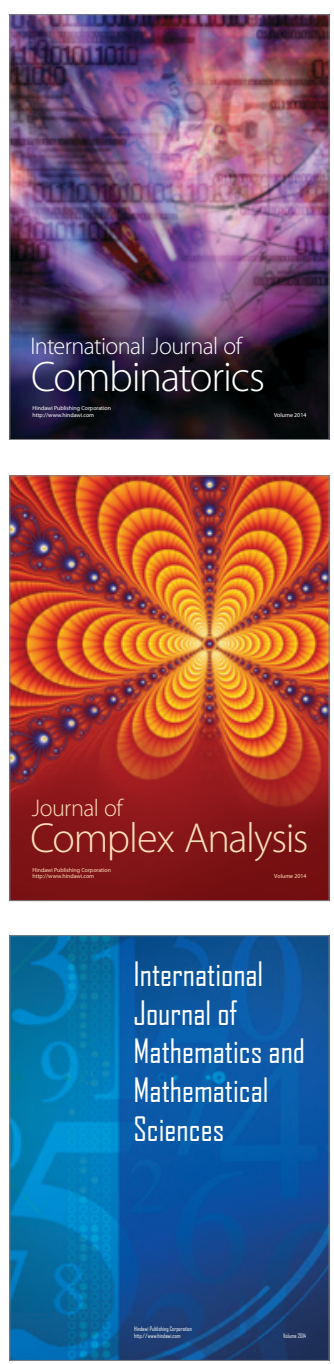
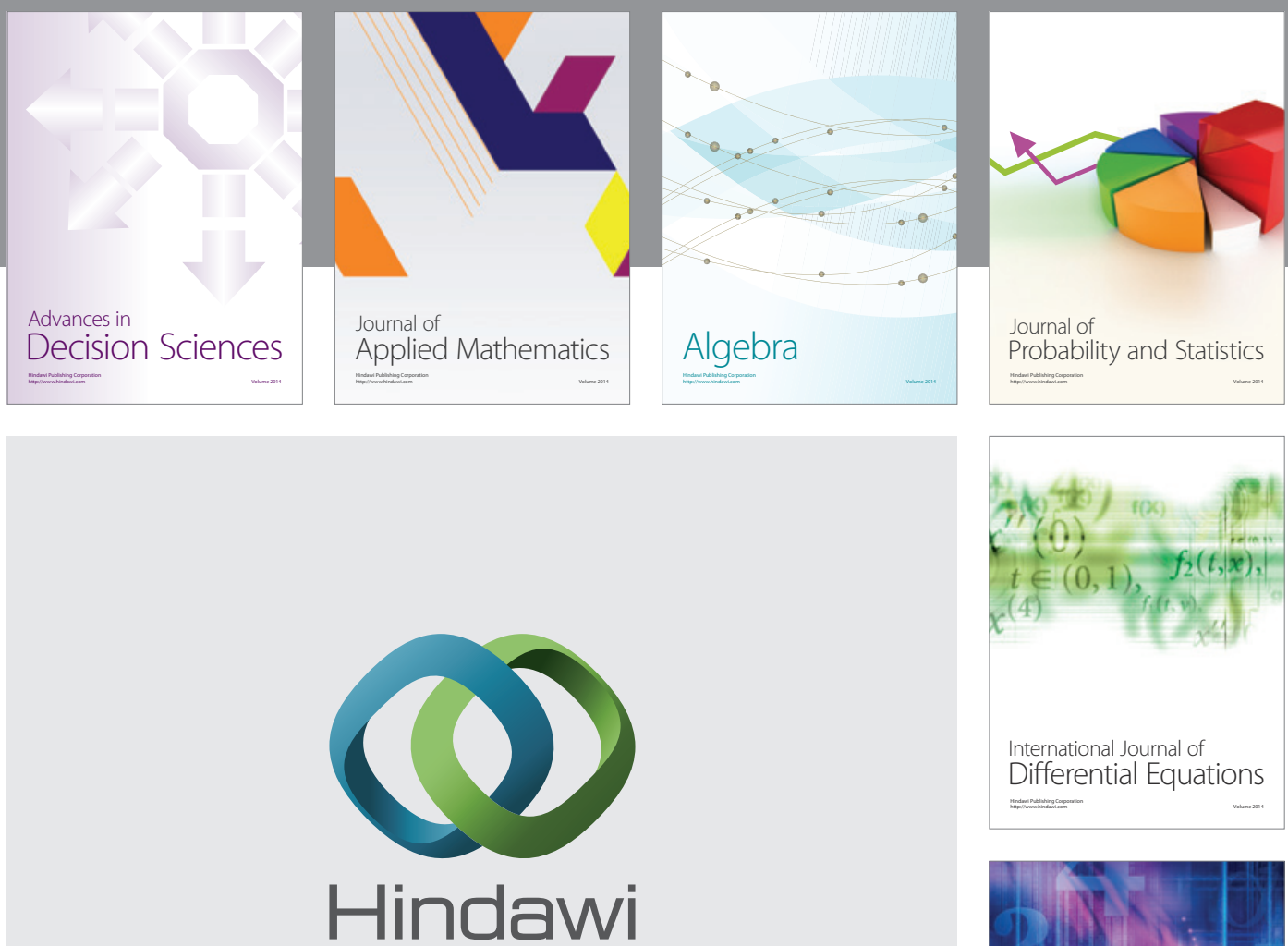

Submit your manuscripts at http://www.hindawi.com
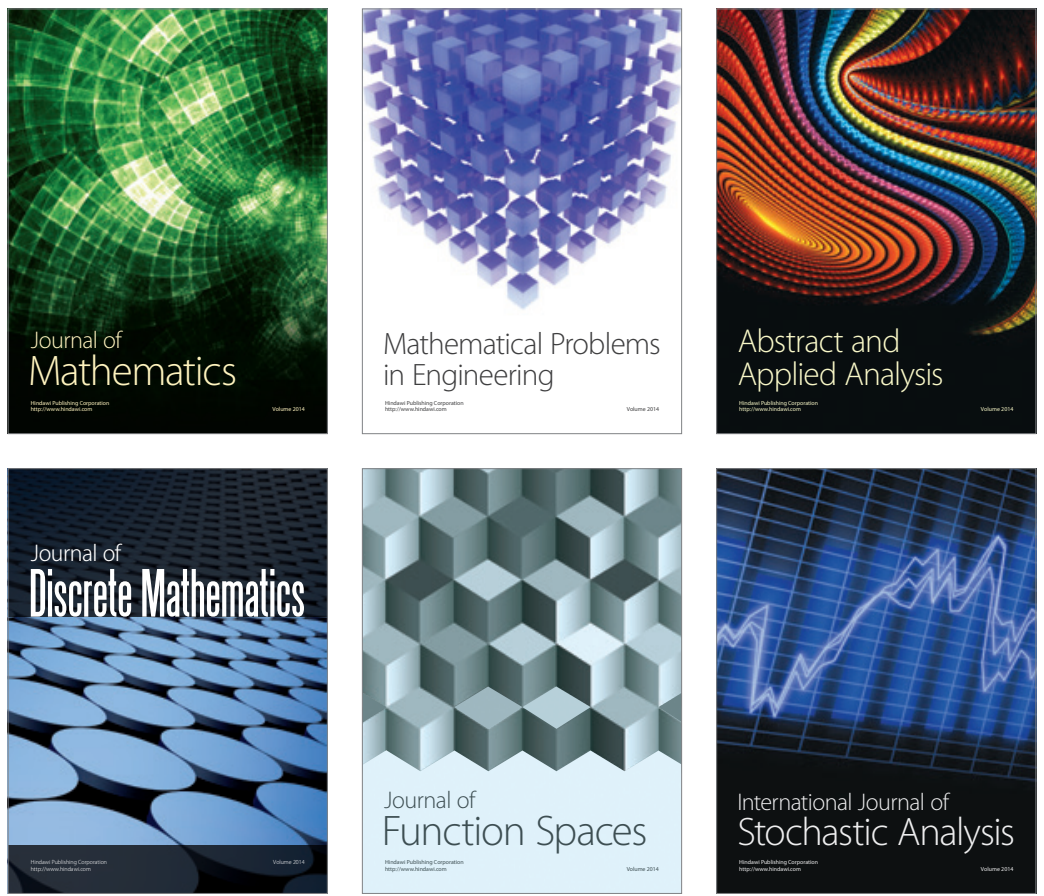

Journal of

Function Spaces

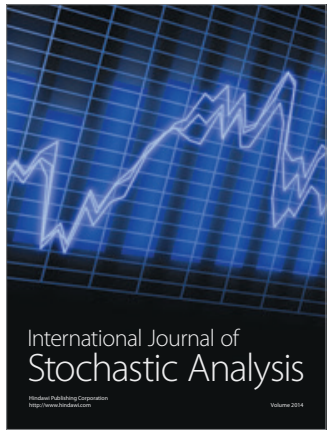

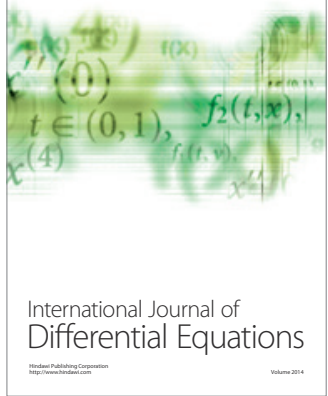
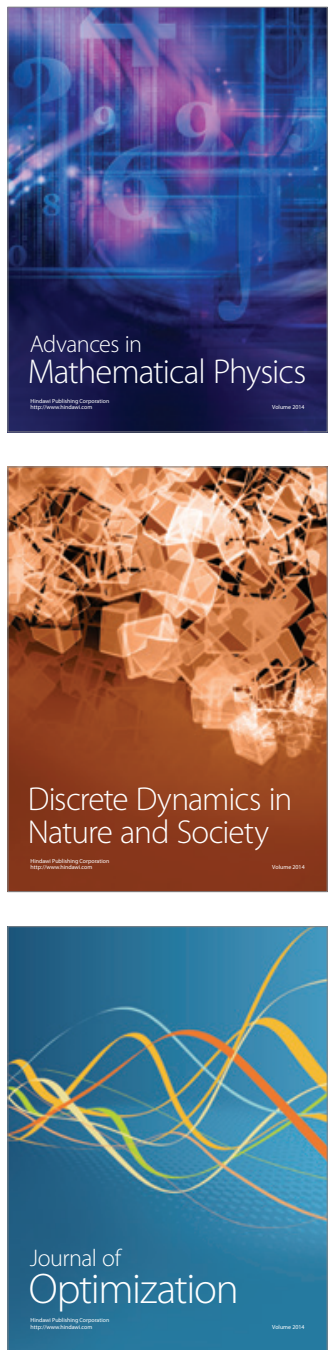